Commun. Korean Math. Soc. 29 (2014), No. 1, pp. 75-81

http://dx.doi.org/10.4134/CKMS.2014.29.1.075

\title{
A SHARP SCHWARZ AND CARATHÉODORY INEQUALITY ON THE BOUNDARY
}

\author{
BÜLENT NAFi ÖRNEK
}

\begin{abstract}
In this paper, a boundary version of the Schwarz and Carathéodory inequality are investigated. New inequalities of the Carathéodory's inequality and Schwarz lemma at boundary are obtained by taking into account zeros of $f(z)$ function which are different from zero. The sharpness of these inequalities is also proved.
\end{abstract}

\section{Introduction}

Let $f$ be a function which is holomorphic on the $D:\{z:|z|<1\}$ and vanish at $z=0$, and suppose that $|f|<1$ for all $z \in D$. Then the inequality

$$
|f(z)| \leq|z|
$$

holds for all $z \in D$, and moreover

$$
\left|f^{\prime}(0)\right| \leq 1
$$

Equality is achieved in (1.1) (for some nonzero $z \in D$ ) or in (1.2) if and only if $f$ is an entire linear function of the form $f(z)=e^{i \alpha} z$, where $\alpha$ is a real number([2], p. 381).

Let the zeros of $f$ be $z_{1}, z_{2}, \ldots, z_{n}$. If we apply inequality (1.1) to the function $f(z) \prod_{k=1}^{n}\left[\frac{1-\overline{z_{k}} z}{z+z_{k}}\right]$, we can conclude in the following Schwarz's inequality:

$$
|f(z)| \leq|z| \prod_{k=1}^{n}\left|\frac{z-z_{k}}{1-\overline{z_{k}} z}\right|
$$

and

$$
\left|f^{\prime}(0)\right| \leq \prod_{k=1}^{n}\left|z_{k}\right|
$$

Received May 3, 2013

2010 Mathematics Subject Classification. Primary 30C80.

Key words and phrases. Schwarz lemma on the boundary, holomorphic function, JuliaWolff-Lemma.

(C)2014 The Korean Mathematical Society 
If $f(z)=c_{p} z^{p}+c_{p+1} z^{p+1}+\cdots, c_{p} \neq 0, p \geq 1$, is a holomorphic function in $D$ and $|f| \leq 1$ for $z \in D$, then at each $z \in D$ we have the inequality

$$
|f(z)| \leq|z|^{p} \prod_{k=1}^{n}\left|\frac{z-z_{k}}{1-\overline{z_{k}} z}\right|
$$

and

$$
\left|c_{p}\right| \leq \prod_{k=1}^{n}\left|z_{k}\right|
$$

If, in addition, the function $f$ can be extended by continuity to a point $z_{0} \in \partial D,\left|f\left(z_{0}\right)\right|=1$, and the derivative $f^{\prime}\left(z_{0}\right)$ exists, then (1.1) implies the inequality $\left|f^{\prime}\left(z_{0}\right)\right| \geq 1$, which is known as the Schwarz lemma on the boundary. Previously, R. Osserman, examined sharp Schwarz inequality at the boundary (see [3]).

If the function $f$ has an angular limit $f\left(z_{0}\right)$ at $z_{0} \in \partial D,\left|f\left(z_{0}\right)\right|=1$, then by Julia-Wolff-Lemma the angular derivative $f^{\prime}\left(z_{0}\right)$ exists and $1 \leq\left|f^{\prime}\left(z_{0}\right)\right| \leq \infty$ $([4])$.

We will obtain more general results at the boundary. In the following Theorems 1.1-1.2, new inequalities of Schwarz inequality at the boundary are obtained and the sharpness of these inequalities is proved.

Introducing the notation

$$
\Phi=\sum_{i_{1}=1}^{n-k+1} \sum_{i_{2}=i_{1}+1}^{n-k+2} \cdots \sum_{i_{k}=i_{k-1}+1}^{n}\left(\left|z_{i_{1}}\right|\left|z_{i_{2}}\right| \cdots\left|z_{i_{k}}\right|\right) .
$$

Theorem 1.1. Let $f$ be a holomorphic function in the disc $D,|f|<1$ for $|z|<1, f(0)=0$ and $z_{1}, z_{2}, \ldots, z_{n}$ are zeros of the function $f$ in the unit disc that are different from $z=0$. Further assume that, for some $z_{0} \in \partial D$, $f$ has an angular limit $f\left(z_{0}\right)$ at $z_{0},\left|f\left(z_{0}\right)\right|=1$. Then

$$
\left|f^{\prime}\left(z_{0}\right)\right| \geq \frac{n+1+\sum_{k=1}^{n}(n-2 k+1) \Phi}{\prod_{k=1}^{n}\left(1+\left|z_{k}\right|\right)} .
$$

The inequality (1.5) is sharp, with equality for the function $f(z)=z \prod_{k=1}^{n} \frac{z-z_{k}}{1-\overline{z_{k}} z}$, where $z_{1}, z_{2}, \ldots, z_{n}$ are negative real numbers.

Proof. Using the upper bound (1.3) and if $z_{0}, c \in \partial D$ with $f\left(z_{0}\right)=c$, then we obtain

$$
\begin{aligned}
& \left|\frac{f(z)-c}{|z|-\left|z_{0}\right|}\right| \geq \frac{1-|f(z)|}{1-|z|} \geq \frac{1-|z| \prod_{k=1}^{n}\left|\frac{z-z_{k}}{1-z_{k} z}\right|}{1-|z|} \geq \frac{1-|z| \prod_{k=1}^{n} \frac{|z|+\left|z_{k}\right|}{1+\left|z_{k}\right||z|}}{1-|z|} \\
= & \frac{\prod_{k=1}^{n}\left(1+\left|z_{k}\right||z|\right)-|z| \prod_{k=1}^{n}\left(|z|+\left|z_{k}\right|\right)}{(1-|z|) \prod_{k=1}^{n}\left(1+\left|z_{k}\right||z|\right)}
\end{aligned}
$$


A SHARP SCHWARZ AND CARATHÉODORY INEQUALITY ON THE BOUNDARY 77

$$
=\frac{1-|z|^{n+1}+\sum_{k=1}^{n}\left(|z|^{k}-|z|^{n-k+1}\right) \sum_{i_{1}=1}^{n-k+1} \sum_{i_{2}=i_{1}+1}^{n-k+2} \ldots i_{i_{k}=i_{k-1}+1}^{n}\left(\left|z_{i_{1}}\right|\left|z_{i_{2}}\right| \cdots\left|z_{i_{k}}\right|\right)}{(1-|z|) \prod_{k=1}^{n}\left(1+\left|z_{k}\right||z|\right)} .
$$

Passing to the angular limit in the last inequality yields

$$
\frac{n+1+\sum_{k=1}^{n}(n-2 k+1) \sum_{i_{1}=1}^{n-k+1} \sum_{i_{2}=i_{1}+1}^{n-k+2} \ldots i_{k}=\sum_{k-1}^{n}\left(\left|z_{i_{1}}\right|\left|z_{i_{2}}\right| \cdots\left|z_{i_{k}}\right|\right)}{\prod_{k=1}^{n}\left(1+\left|z_{k}\right|\right)} .
$$

The equality in (1.5) is obtained for the function $f(z)=z \prod_{k=1}^{n} \frac{z-z_{k}}{1-\overline{z_{k}} z}$, as show simple calculations.

Theorem 1.2. Let $f(z)=c_{p} z^{p}+c_{p+1} z^{p+1}+\cdots, c_{p} \neq 0, p \geq 1$, be a holomorphic function in the disc $D,|f|<1$ for $|z|<1$ and $z_{1}, z_{2}, \ldots, z_{n}$ are zeros of the function $f$ in the unit disc that are different from $z=0$. Further assume that, for some $z_{0} \in \partial D, f$ has an angular limit $f\left(z_{0}\right)$ at $z_{0},\left|f\left(z_{0}\right)\right|=1$. Then

$$
\left|f^{\prime}\left(z_{0}\right)\right| \geq \frac{n+p+\sum_{k=1}^{n}(n-2 k+p) \Phi}{\prod_{k=1}^{n}\left(1+\left|z_{k}\right|\right)}
$$

The inequality (1.6) is sharp, with equality for the function

$$
f(z)=z^{p} \prod_{k=1}^{n} \frac{z-z_{k}}{1-\overline{z_{k}} z},
$$

where $z_{1}, z_{2}, \ldots, z_{n}$ are negative real numbers.

Proof. Using the upper bound (1.4) and if $z_{0}, c \in \partial D$ with $f\left(z_{0}\right)=c$, then we obtain

$$
\begin{aligned}
& \left|\frac{f(z)-c}{|z|-\left|z_{0}\right|}\right| \geq \frac{1-|f(z)|}{1-|z|} \geq \frac{1-|z|^{p} \prod_{k=1}^{n}\left|\frac{z-z_{k}}{1-\bar{z}_{k} z}\right|}{1-|z|} \geq \frac{1-|z|^{p} \prod_{k=1}^{n} \frac{|z|+\left|z_{k}\right|}{1-|z| z_{k}|| z \mid}}{1-|z|} \\
= & \frac{\prod_{k=1}^{n}\left(1+\left|z_{k}\right||z|\right)-|z|^{p} \prod_{k=1}^{n}\left(|z|+\left|z_{k}\right|\right)}{(1-|z|) \prod_{k=1}^{n}\left(1+\left|z_{k}\right||z|\right)} \\
= & \frac{1-|z|^{n+p}+\sum_{k=1}^{n}\left(|z|^{k}-|z|^{n-k+p}\right) \sum_{i_{1}=1}^{n-k+1} i_{i_{2}=i_{1}+1}^{n-k+2} \ldots}{(1-|z|) \prod_{k=1}^{n}\left(1+\left|z_{k}\right||z|\right)} \sum_{i_{k-1}}^{n}\left(\left|z_{i_{1}}\right|\left|z_{i_{2}}\right| \cdots\left|z_{i_{k}}\right|\right)
\end{aligned}
$$

Passing to the angular limit in the last inequality yields

$$
\frac{n+p+\sum_{k=1}^{n}(n-2 k+p) \sum_{i_{1}=1}^{n-k+1} \sum_{i_{2}=i_{1}+1}^{n-k+2} \ldots i_{k}=\sum_{k-1}^{n}\left(\left|z_{i_{1}}\right|\left|z_{i_{2}}\right| \cdots\left|z_{i_{k}}\right|\right)}{\prod_{k=1}^{n}\left(1+\left|z_{k}\right|\right)} .
$$


The equality in (1.6) is obtained for the function $f(z)=z^{p} \prod_{k=1}^{n} \frac{z-z_{k}}{1-\overline{z_{k}} z}$, as show simple calculations.

In the following theorems, we formulated boundary "Carathéodory inequality" (see [1]) as long the Schwarz lemma at the boundary (see [3]). Besides, we have following results, which can be offered as the boundary refinement of the Carathéodory inequality.

Theorem 1.3. Let $f$ be a holomorphic function in the disc $D, f(0)=0$ and $z_{1}, z_{2}, \ldots, z_{n}$ are zeros of the function $f$ in the unit disc that are different from $z=0$. Let $\Re f \leqslant A$ for $|z|<1$. Further assume that, for some $z_{0} \in \partial D$, $f$ has an angular limit $f\left(z_{0}\right)$ at $z_{0}, \Re f\left(z_{0}\right)=A$. Then

$$
\left|f^{\prime}\left(z_{0}\right)\right| \geq \frac{A}{2} \frac{n+1+\sum_{k=1}^{n}(n-2 k+1) \Phi}{\prod_{k=1}^{n}\left(1+\left|z_{k}\right|\right)} .
$$

The inequality (1.7) is sharp, with equality for the function

$$
f(z)=\frac{2 A z \prod_{k=1}^{n} \frac{z-z_{k}}{1-\overline{z_{k}} z}}{1+z \prod_{k=1}^{n} \frac{z-z_{k}}{1-\bar{z}_{k} z}}
$$

where $z_{1}, z_{2}, \ldots, z_{n}$ are negative real numbers.

Proof. Consider the function

$$
w(z)=\frac{f(z)}{f(z)-2 A},|z|<1 .
$$

Since $\Re f\left(z_{0}\right)=A,\left|w\left(z_{0}\right)\right|=1$ for $z_{0} \in \partial D$ and the function $w(z)$ is defined in (1.8) satisfies the assumptions of Theorem 1.1, we obtain

$$
\left|w^{\prime}\left(z_{0}\right)\right| \geq \frac{n+1+\sum_{k=1}^{n}(n-2 k+1) \Phi}{\prod_{k=1}^{n}\left(1+\left|z_{k}\right|\right)}
$$

and

$$
\frac{2 A\left|f^{\prime}\left(z_{0}\right)\right|}{\left|f\left(z_{0}\right)-2 A\right|^{2}} \geq \frac{n+1+\sum_{k=1}^{n}(n-2 k+1) \Phi}{\prod_{k=1}^{n}\left(1+\left|z_{k}\right|\right)} .
$$

Since $\left|f\left(z_{0}\right)-2 A\right|^{2} \geq\left[\Re\left(f\left(z_{0}\right)-2 A\right)\right]^{2}=A^{2}$, we obtain inequality (1.7) with an obvious equality case.

Theorem 1.4. Let $f(z)=c_{p} z^{p}+c_{p+1} z^{p+1}+\cdots, c_{p} \neq 0, p \geq 1$, be a holomorphic function in the disc $D$ and $z_{1}, z_{2}, \ldots, z_{n}$ are zeros of the function $f$ in the 
unit disc that are different from $z=0$. Let $\Re f \leqslant A$ for $|z|<1$. Further assume that, for some $z_{0} \in \partial D, f$ has an angular limit $f\left(z_{0}\right)$ at $z_{0}, \Re f\left(z_{0}\right)=A$. Then

$$
\left|f^{\prime}\left(z_{0}\right)\right| \geq \frac{A}{2} \frac{n+p+\sum_{k=1}^{n}(n-2 k+p) \Phi}{\prod_{k=1}^{n}\left(1+\left|z_{k}\right|\right)} .
$$

The inequality (1.9) is sharp, with equality for the function

$$
f(z)=\frac{2 A z^{p} \prod_{k=1}^{n} \frac{z-z_{k}}{1-\bar{z}_{k} z}}{1+z^{p} \prod_{k=1}^{n} \frac{z-z_{k}}{1-\overline{z_{k}} z}}
$$

where $z_{1}, z_{2}, \ldots, z_{n}$ are negative real numbers.

Proof. The function $w(z)$ is defined in (1.8) satisfies the assumptions of Theorem 1.2 , we obtain

and

$$
\left|w^{\prime}\left(z_{0}\right)\right| \geq \frac{n+p+\sum_{k=1}^{n}(n-2 k+p) \Phi}{\prod_{k=1}^{n}\left(1+\left|z_{k}\right|\right)}
$$

$$
\frac{2 A\left|f^{\prime}\left(z_{0}\right)\right|}{\left|f\left(z_{0}\right)-2 A\right|^{2}} \geq \frac{n+p+\sum_{k=1}^{n}(n-2 k+p) \Phi}{\prod_{k=1}^{n}\left(1+\left|z_{k}\right|\right)} .
$$

Since $\left|f\left(z_{0}\right)-2 A\right|^{2} \geq\left[\Re\left(f\left(z_{0}\right)-2 A\right)\right]^{2}=A^{2}$, we obtain inequality (1.9) with an obvious equality case.

Theorem 1.5. Let $f$ be a holomorphic function in the disc $D$ and $z_{1}, z_{2}, \ldots, z_{n}$ are zeros of the function $f(z)-f(0)$ in the unit disc that are different from $z=0$. Let $\Re f \leqslant A$ for $|z|<1$. Further assume that, for some $z_{0} \in \partial D$, f has an angular limit $f\left(z_{0}\right)$ at $z_{0}, \Re f\left(z_{0}\right)=A$. Then

$$
\left|f^{\prime}\left(z_{0}\right)\right| \geq\left(\frac{A-\Re f(0)}{2}\right) \frac{n+1+\sum_{k=1}^{n}(n-2 k+1) \Phi}{\prod_{k=1}^{n}\left(1+\left|z_{k}\right|\right)} .
$$

The inequality (1.10) is sharp, with equality for the function

$$
f(z)=f(0)+\frac{2(A-\Re f(0)) z \prod_{k=1}^{n} \frac{z-z_{k}}{1-\overline{z_{k}} z}}{1+z \prod_{k=1}^{n} \frac{z-z_{k}}{1-\overline{z_{k}} z}}
$$

where $z_{1}, z_{2}, \ldots, z_{n}$ are negative real numbers.

Proof. Introducing the notation

$$
\alpha=A-\Re f(z), \beta=A-\Re f(0) .
$$

If $f$ is not identically constant, then $\alpha>0, \beta>0, \Re(f(z)-f(0))=\beta-\alpha$ $<\beta$ and $4 \beta \Re(f(z)-f(0)) \leq 4 \beta^{2}$. Therefore

$|f(z)-f(0)-2 \beta|^{2}=|f(z)-f(0)|^{2}-4 \beta \Re(f(z)-f(0))+4 \beta^{2}>|f(z)-f(0)|^{2}$. 
The function

$$
\varphi(z)=\frac{f(z)-f(0)}{f(z)-f(0)-2 \beta}
$$

is a holomorphic function in the $\operatorname{disc} D,|\varphi(z)|<1, \varphi(0)=0$ and $\left|\varphi\left(z_{0}\right)\right|=1$ for $z_{0} \in \partial D$. The function $\varphi(z)$ satisfies the assumptions of Theorem 1.1, we obtain

$$
\left|\varphi^{\prime}\left(z_{0}\right)\right| \geq \frac{n+1+\sum_{k=1}^{n}(n-2 k+1) \sum_{i_{1}=1}^{n-k+1} \sum_{i_{2}=i_{1}+1}^{n-k+2} \ldots \sum_{i_{k}=i_{k-1}+1}^{n}\left(\left|z_{i_{1}}\right|\left|z_{i_{2}}\right| \cdots\left|z_{i_{k}}\right|\right)}{\prod_{k=1}^{n}\left(1+\left|z_{k}\right|\right)} .
$$

Therefore, we take

$$
\frac{2 \beta\left|f^{\prime}\left(z_{0}\right)\right|}{|f(z)-f(0)-2 \beta|^{2}} \geq \frac{n+1+\sum_{k=1}^{n}(n-2 k+1) \Phi}{\prod_{k=1}^{n}\left(1+\left|z_{k}\right|\right)} .
$$

Since $\left|f\left(z_{0}\right)-f(0)\right|^{2} \geq\left[\Re\left(f\left(z_{0}\right)-f(0)\right)\right]^{2}=\beta^{2}$, we obtain inequality (1.10) with an obvious equality case.

Theorem 1.6. Let $f(z)=f(0)+c_{p} z^{p}+c_{p+1} z^{p+1}+\cdots, c_{p} \neq 0, p \geq 1$, be a holomorphic function in the disc $D$ and $z_{1}, z_{2}, \ldots, z_{n}$ are zeros of the function $f(z)-f(0)$ in the unit disc that are different from $z=0$. Let $\Re f \leqslant A$ for $|z|<1$. Further assume that, for some $z_{0} \in \partial D, f$ has an angular limit $f\left(z_{0}\right)$ at $z_{0}, \Re f\left(z_{0}\right)=A$. Then

$$
\left|f^{\prime}\left(z_{0}\right)\right| \geq\left(\frac{A-\Re f(0)}{2}\right) \frac{n+p+\sum_{k=1}^{n}(n-2 k+p) \Phi}{\prod_{k=1}^{n}\left(1+\left|z_{k}\right|\right)} .
$$

The inequality (1.11) is sharp, with equality for the function

$$
f(z)=f(0)+\frac{2(A-\Re f(0)) z^{p} \prod_{k=1}^{n} \frac{z-z_{k}}{1-\bar{z}_{k} z}}{1+z^{p} \prod_{k=1}^{n} \frac{z-z_{k}}{1-\bar{z}_{k} z}},
$$

where $z_{1}, z_{2}, \ldots, z_{n}$ are negative real numbers.

Proof. The function $\varphi(z)$ is defined in Theorem 1.5 satisfies the assumptions of Theorem 1.4, we obtain

$$
\left|\varphi^{\prime}\left(z_{0}\right)\right| \geq \frac{n+p+\sum_{k=1}^{n}(n-2 k+p) \Phi}{\prod_{k=1}^{n}\left(1+\left|z_{k}\right|\right)} .
$$

Therefore, we take

$$
\frac{2 \beta\left|f^{\prime}\left(z_{0}\right)\right|}{|f(z)-f(0)-2 \beta|^{2}} \geq \frac{n+p+\sum_{k=1}^{n}(n-2 k+p) \Phi}{\prod_{k=1}^{n}\left(1+\left|z_{k}\right|\right)} .
$$

Since $\left|f\left(z_{0}\right)-f(0)\right|^{2} \geq\left[\Re\left(f\left(z_{0}\right)-f(0)\right)\right]^{2}=\beta^{2}$, we obtain inequality (1.11) with an obvious equality case. 
A SHARP SCHWARZ AND CARATHÉODORY INEQUALITY ON THE BOUNDARY 81

\section{References}

[1] G. Kresin and V. Maz'ya, Sharp Real-Part Theorems: A Unified Approach, Translated from the Russian and edited by T. Shaposhnikova. Lecture Notes in Mathematics, 1903. Springer, Berlin, 2007.

[2] A. I. Markushevich, Theory of Functions of a Complex Variable. Vol. I, Prentice-Hall, Inc., Englewood Cliffs, N.J. 1965.

[3] R. Osserman, A sharp Schwarz inequality on the boundary, Proc. Amer. Math. Soc. 128 (2000), no. 12, 3513-3517.

[4] Ch. Pommerenke, Boundary Behaviour of Conformal Maps, Springer-Verlag, Berlin, 1992.

Department of Mathematics

Gebze Institute of Technology

Gebze-KocAeli 41400, TuRKey

E-mail address: nornek@gyte.edu.tr 